\title{
CIEN AÑOS DE MULTILATERALISMO Y RETOS PARA EL SEGUNDO CENTENARIO
}

\section{Oscar Schiappa-Pietra Cubas *}

Agradezco a la Embajada de Suiza, al Instituto de Estudios Social Cristianos y a la Sociedad Peruana de Derecho Internacional por organizar este evento e invitarme como expositor.

Es especialmente significativo que estemos ahora congregados bajo la hospitalidad del Gobierno de Suiza, pues este país cumple un rol muy significativo promoviendo el multilateralismo. Ginebra, es reconocida como la capital mundial del multilateralismo, pues fue sede de la Sociedad de Naciones, y actualmente alberga a las sedes de alrededor de cuarenta organismos internacionales, incluyendo la sede europea de las Naciones Unidas, y de más de setecientas organizaciones no-gubernamentales.

El 8 de enero de 1918, precisamente cuando las tropas estadounidenses empezaban a revertir la correlación bélica de la Primer Guerra Mundial, el presidente Woodrow Wilson enunció sus propuestas para forjar un escenario internacional con vocación de paz, los célebres 14 puntos. En estos se plantearon fórmulas para zanjar diversas disputas territoriales y responder a las aspiraciones nacionales, en Europa; se abogó por acabar con el secretismo en las negociaciones y los acuerdos diplomáticos; se enunció la necesidad de promover el libre comercio, el desarme y la autodeterminación de los pueblos; y, en el último punto, se propuso "[Que u]na asociación general de naciones debe ser formada bajo acuerdos específicos con el propósito de otorgar garantías mutuas de independencia política e integridad territorial, a estados grandes y pequeños por igual".

Con algo de ironía y escepticismo, pero sin contradecir el acierto de los 14 puntos de Wilson, el Primer Ministro francés, George Clemenceau, señaló: "Dios nos dio los Diez Mandamientos y los quebrantamos. Wilson nos da los Catorce Puntos. Veremos."

El revolucionario planteamiento contenido en el rubro final de los 14 puntos del presidente Wilson encontró eco: hace un siglo y pocas semanas más, el 28 de junio de 1919, se suscribió el Tratado de Versalles, que formalmente puso fin a la terrible devastación de la Primera Guerra Mundial, y que -a través de su parte primera, consagrada al establecimiento de la Sociedad de Naciones- sentó los cimientos pioneros del orden multilateral de gobernanza global que hemos heredado.

Esa guerra, y su transacción a través del Tratado de Versalles, transformaron radicalmente el mapa geopolítico del mundo. La impronta de este acuerdo internacional, particularmente en lo que respecta a su más importante hito -la creación de la Sociedad

\footnotetext{
* Conferencia en el Conversatorio A Cien Años de la Creación de la Sociedad de Naciones. Retos y Oportunidades del Multilateralismo. Evento organizado por la Sociedad Peruana de Derecho Internacional (SPDI), la Embajada de Suiza en el Perú y el Instituto de Estudios Social Cristianos (IESC), el lunes 02 de diciembre de 2019
} 
de Naciones- ha tenido enorme impacto durante el siglo XX, y sigue manteniéndolo hasta la actualidad.

Pero, antes de continuar, cabe reflexionar sobre por qué es importante el tema que nos convoca hoy. Lo es:

- porque el reto de la sostenibilidad humana se nutre de las promesas de convivencia pacífica, cooperación y solidaridad internacionales, democracia y derechos humanos, que son los postulados fundamentales del multilateralismo;

- porque los complejos problemas que afrontamos como especie humana y habitantes del Hogar Común, que es nuestro planeta, requieren de respuestas desde la gobernanza global que escapan a las competencias y capacidades de los estados aisladamente;

- y, porque el multilateralismo es el más reciente pilar del sistema internacional que la humanidad ha venido forjando a lo largo de los últimos cuatro siglos.

Como sabemos, se atribuye -algo imprecisamente- el origen del actual sistema de organización jurídico-política internacional a los dos tratados de paz de Osnabrück y Münster, firmados el 15 de mayo y 24 de octubre de 1648, es decir, a lo que comúnmente se denomina la Paz de Westfalia. Las premisas fundamentales del marco conceptual y ordenamiento político westfaliano son la preeminencia del Estado como actor internacional, su atributo de soberanía estatal, y la territorialidad.

Si bien esa racionalidad westfaliana posibilitó eliminar la conflictividad bélica generada por diferencias religiosas en el espacio europeo del siglo XVII, fue incapaz de crear condiciones de paz generalmente sostenibles.

Ya a través de la Primera Guerra Mundial, la humanidad se vio confrontada con su desbocada capacidad de destrucción, catalizada, aún de modo más brutal que antes, por las innovaciones que la revolución industrial introdujo en la tecnología militar. En esa malhadada confrontación, más de diez millones de vidas se perdieron, los daños materiales fueron cuantiosísimos, y cuatro imperios se extinguieron.

La comunidad internacional de inicios del siglo XX cayó en cuenta sobre su propia inhabilidad para contener las barbaries bélicas apelando exclusivamente a la voluntad soberana de los estados. Así pareció reconocerlo el mismo Primer Ministro George Clemenceau, cuando el 11 de noviembre de 1918, día de la firma del armisticio de la Primera Guerra Mundial, sostuvo: "Hemos ganado la guerra, y no sin dolor; ahora habrá que ganar la paz, y esto seguramente será más difícil”.

Los estados firmantes del Tratado de Versalles se comprometieron a forjar un orden internacional que dejara atrás la confrontación como impulso fundamental en las relaciones entre ellos, para dar paso a la cooperación y a la confianza mutua como nueva gramática de las relaciones internacionales. Con la participación de los países europeos y Estados Unidos, secundados por diecisiete de los veinte países latinoamericanos, se fundó la Sociedad de Naciones.

La eficacia de este pionero emprendimiento multilateral requiere ser evaluada en dos dimensiones temporales distintas. De un lado, en lo históricamente inmediato, la Sociedad de Naciones fracasó en crear condiciones políticas para una paz sostenible, y en otras áreas solo alcanzó limitadísima eficacia. El historiador Edward Hallett Carr, sostiene que este pionero proyecto multilateral adoleció por abrigar un idealismo 
arraigado en el liberalismo decimonónico, y que es aquí -en sus principios fundacionalesdonde estuvo la causa de su ineficacia. Según el mismo Carr, el yerro no radicó en los individuos; ni en el cambio en la postura de los Estados Unidos, cuyo Senado no aprobó la membresía de su país en la Sociedad de Naciones, pese a que su fundación estuvo inmensamente inspirada en la visión y el liderazgo del presidente Wilson.

La precaria paz internacional alcanzada a través del Tratado de Versalles y de la Sociedad de Naciones colapsó prontamente, teniendo como gran catalizador al caos económico internacional que signó la década de 1930. Este organismo fue incapaz de contener las ambiciones de potencias revisionistas en Europa y Asia, o de amenguar el espíritu de profunda humillación de Alemania.

En particular, las severísimas condiciones impuestas en el Tratado de Versalles a Alemania, como perdedora de la Primera Guerra Mundial, sirvieron como combustible para propagar el resentimiento dentro de su sociedad hacia el resto de la comunidad internacional, propiciando el surgimiento del nazismo y de su protagonismo en el desencadenamiento de la Segunda Guerra Mundial. Ya John Maynard Keynes, en su libro de 1919, Las consecuencias económicas de la paz, había llamado la atención sobre lo excesivamente gravosa de la obligación de reparaciones impuesta a Alemania por el Tratado de Versalles, augurando que ello originaría una nueva crisis europea.

Pese a sus evidentes limitaciones como organismo encargado de preservar la paz internacional, la Sociedad de Naciones alcanzó moderado éxito en lograr estabilizar la situación de nuevos estados; en proteger a minorías; y en facilitar la transformación formal de territorios colonizados en estados soberanos.

Pero, de otro lado, analizado el rol de la Sociedad de Naciones desde una perspectiva de largo plazo, es menester reconocer que sembró las semillas de la cultura y la práctica del multilateralismo, y que sirvió de inspirador referente para la formación de las Naciones Unidas, al acabar la Segunda Guerra Mundial. Especialmente relevante es la consagración de la paz como un objetivo global en el Tratado de Versalles y su implementación a través de la Sociedad de Naciones, a través de la pionera noción de seguridad colectiva. El artículo $11.1^{\circ}$ del Tratado de Versalles estipuló: "Se declara expresamente que toda guerra o amenaza de guerra, afecte directamente o no a uno de los miembros de la sociedad, interesa a la sociedad entera y que ésta debe adoptar las medidas adecuadas para salvaguardar eficazmente la paz de las naciones." Y el artículo $10^{\circ}$ consagró el principio de soberanía de los estados: "Los miembros de la sociedad se comprometen a respetar y a mantener contra toda agresión exterior la integridad territorial y la independencia política presente de todos los miembros de la sociedad."

En torno a la Sociedad de Naciones se establecieron una gran cantidad de comités, institutos y organizaciones a cargo de variados asuntos económicos, sociales, humanitarios y culturales. Entidades como la Organización Internacional del Trabajo, la Organización Mundial de la Salud, la UNESCO y el UNICEF, emergieron de la estructura institucional forjada por la Sociedad de Naciones, y cumplen hasta nuestros días un rol vital de cooperación internacional especializada. Mención especial merece la Corte Internacional de Justicia, cuya antecesora también fue parte del sistema de la Liga de Naciones. 
La Segunda Guerra Mundial ha sido la confrontación bélica más destructiva en la historia de la humanidad: un estimado de 70 a 85 millones de personas, es decir alrededor de $3 \%$ de la población mundial perdieron la vida, muchísimas de modos extremadamente crueles; Europa y Japón quedaron materialmente devastadas. Sobre esas cenizas, se forjó en 1945 las Naciones Unidas.

Los países vencedores de la Segunda Guerra Mundial prestaron especial atención en evitar repetir el principal error del Tratado de Versalles. Estados Unidos, en particular, estableció en 1948 un programa de asistencia masiva para la reconstrucción de Europa, el Plan Marshall, destinando el $11 \%$ de sus fondos para financiar la rehabilitación de Alemania Occidental.

En la Carta de las Naciones Unidas se reafirman los principios básicos de paz y cooperación internacional a través de la coexistencia pacífica entre estados; se reconoce la dignidad inherente a toda persona, condensada en el concepto de derechos humanos; se reitera el principio de seguridad colectiva, mejorando los mecanismos para su implementación y se establece, a través de su artículo $42^{\circ}$, la atribución de la Organización para actuar militarmente contra estados que pongan en peligro la paz mundial.

Las Naciones Unidas puede reivindicar el haber logrado la membrecía en su seno de la totalidad de los estados soberanos; su rol promotor de los derechos humanos y de los valores democráticos; su eficacia en guiar el proceso de descolonización a nivel global; su soporte en la afirmación y desarrollo del derecho internacional; y su liderazgo en la institucionalización de la cooperación internacional.

A la vez -hay que reconocerlo- la Carta de las Naciones Unidas consagra una estructura de gobernanza global defectuosa, particularmente en lo concerniente a su Consejo de Seguridad. A través de su artículo $27.3^{\circ}$, la Carta otorga a cinco naciones, vencedoras en la Segunda Guerra Mundial, el privilegio de ejercer un derecho de veto, que contradice el principio de igualdad soberana de los estados, consagrado en el art. $2.1^{\circ}$ de la misma Carta. Además de la disfuncionalidad históricamente aparejada por el ejercicio de tal derecho de veto, este órgano ejecutivo fundamental carece de sometimiento a elementales principios democráticos y expresa con gran crudeza el dominio hegemónico de las grandes potencias.

Y es que las Naciones Unidas no puede sustraerse de las dinámicas políticas de su tiempo. Su funcionamiento quedó reducido a la ineficacia en asuntos de seguridad internacional que afectaban intereses estratégicos de los dos hegemones del orden bipolar, como ha quedado sistemáticamente evidenciado en el desenvolvimiento del Consejo de Seguridad de las Naciones Unidas.

No obstante ello, hoy, coexistimos en un mundo más gobernable del que existía hace tres cuartos de siglo, teniendo la paz, la dignidad y bienestar personales, y el progreso social, inmensas mayores oportunidades de realización. A través de los 17 Objetivos de Desarrollo Sostenibles, la comunidad internacional comparte ahora una estrategia común para enfrentar algunos de los principales desafíos contemporáneos. Y, como lo ha anotado John Ruggie, el orden multilateral liderado por las Naciones Unidas ha posibilitado estabilizar las consecuencias generadas por la disolución de la bipolaridad. 
Pero hay que decirlo con claridad: es igualmente cierto que los problemas que hoy afrontamos como humanidad vienen desbordando las capacidades de nuestros gobernantes, de nuestros diplomáticos y políticos, y de los organismos internacionales, para darles cara. Estamos dejando a las futuras generaciones un cúmulo de problemas sin resolver y de riesgos que demandan urgente atención. De cara a estos inmensos desafíos, necesitamos reflexionar sobre el nuevo perfil de multilateralismo que las realidades contemporáneas y futuras demandan.

Hacia tal rumbo, resulta fundamental dejar anotadas las debilidades del actual sistema multilateral. Una primera es el llamado déficit democrático. Cada vez más, las decisiones fundamentales sobre temas que afectan el bienestar y comprometen el futuro de cada uno de nosotros, los ciudadanos del mundo, vienen dejando de ser adoptadas en la esfera interna de cada Estado, y se convierten en competencia de instancias internacionales, públicas o privadas. Mientras todos elegimos mediante el voto a nuestras autoridades políticas nacionales, e influimos por diversos medios en las decisiones que ellas adoptan; nada parecido ocurre en la esfera internacional, donde muchísimas vitales decisiones son acordadas con poca transparencia, alejadas de medios de participación y de control ciudadanos, y sin que nuestras voces, intereses y puntos de vista sean tenidos en cuenta. Las tendencias globalizadoras acentúan cada vez más este déficit democrático. Las protestas sociales que en estos días asolan a muy diversos países en el mundo expresan el descontento popular ante esta progresiva erosión de los atributos ciudadanos.

Y hay que tomar conciencia que poco podrá avanzarse en superar este déficit democrático en la esfera multilateral si a la misma vez existe una notoria regresión en la observancia de los estándares de derechos humanos y de democracia por parte de los estados en el ámbito nacional. De un lado están los países sometidos a regímenes autoritarios, que vienen cobrando mayor protagonismo en el concierto de las naciones; pero, del otro, está la preocupante fatiga democrática y la pérdida de convicción en los dirigentes políticos y ciudadanos de los propios países que históricamente eran la vanguardia en la observancia y promoción de los valores de la libertad y la dignidad humanas.

La segunda área de debilidad del sistema multilateral está referida a su carácter descentralizado, y a la multiplicación y enorme diversificación que venimos experimentando en el universo de los actores internacionales. Esto dificulta la forja de consensos, y viene generando gran fragmentación en los mecanismos de gobernanza global, dada la poca coordinación existente entre ellos, al par que se multiplican las fuentes normativas, incluyendo la diversificación de las instancias judiciales internacionales. Tal fragmentación dificulta la capacidad para generar respuestas eficaces frente a desafíos que demandan soluciones holísticas y genuinamente globales.

La tercera área de debilidad la encontramos en la falta de consensos, que se expresa también en la ausencia de expresiones institucionales, para regular aspectos críticos de la convivencia y la sostenibilidad globales. Por ejemplo: el ciberespacio es hoy una dimensión fundamental de nuestras vidas, de cómo nos relacionamos con los demás, de cómo nos comunicamos e informamos, de cómo comerciamos. No obstante su centralidad en el quehacer cotidiano de la humanidad, el ciberespacio carece de mecanismos mínimamente eficaces para su gobernabilidad global. Esto viene generando inmensas distorsiones y nuevos riesgos, a través de la concentración monopólica privada 
a escala global por parte de unos pocos conglomerados, o de la masiva erosión de la privacidad, o del surgimiento de graves amenazas de ciberseguridad.

Peor aún, viene intensificándose el riesgo que el ciberespacio se convierta en un nuevo escenario en la creciente confrontación entre China y los Estados Unidos, generando así fragmentación en sus estructuras tecnológicas, haciéndolo vehículo de mutuas agresiones, y despojándolo de su vocación de libertad que tanto viene contribuyendo a ensanchar las fronteras de la creatividad humana. Thomas L. Friedman acaba de referirse a esto: "Pero nadie parecía darse cuenta que casi exactamente 30 años luego de la caída del Muro de Berlín, un nuevo muro -un Muro de Berlín digital- había empezado a construirse entre China y los Estados Unidos”. (Friedman, 2019).

Esta misma debilidad se proyecta, asimismo, en otras muchas esferas, como las de la gestión de la sostenibilidad medioambiental de nuestro Hogar Común; y de la lucha contra la criminalidad trasnacional, la corrupción y el lavado de dinero. En todos esos casos, las respuestas institucionales para su gobernanza multilateral son precarias e insuficientes.

De otro lado, como ya ha quedado señalado, el desenvolvimiento del multilateralismo está determinado por la realidad política global. Ahora estamos atravesando un proceso de profundos cambios, cuya evolución hacia el mediano plazo resulta incierta. Casi toda la segunda mitad del siglo XX estuvo signada por la competencia bipolar, que dio luego paso a una temporalmente efímera unipolaridad, y que en estos días va transformándose hacia la multipolaridad. La consiguiente competencia por hegemonía entre tres grandes potencias -Estados Unidos, China y en menor medida Rusia- torna muy complejo el escenario dentro del cual el multilateralismo tiene que redefinirse.

Todas estas reflexiones tienen un significado especial en estos días, cuando los ciudadanos en muy diversos países toman las calles para protestar. Es demasiado temprano para formular diagnósticos exhaustivos sobre sus motivaciones, pero en un nivel epidérmico es evidente que lo que esos hombres y mujeres de muy diversas latitudes están expresando son sus insatisfacciones con el desenvolvimiento de la democracia, tanto en sus dimensiones nacionales como multilaterales; de la globalización y de la gobernanza global. Ese descontento ciudadano no constituye novedad: este último fin de semana se han cumplido veinte años de las protestas contra la globalización que tuvieron lugar en Seattle, con ocasión de la Reunión Ministerial de la Organización Mundial del Comercio. Es pertinente recordarlo porque el funcionamiento de la OMC grafica nítidamente las virtudes pero también los defectos del multilateralismo, que es catalista de la globalización.

La OMC ha asumido, sin mayor control democrático y sin contar con el consenso de todos sus estados miembros, funciones regulatorias en una gran diversidad de áreas que en puridad no son de comercio internacional, y se ha tornado en promotora de la denominada hiperglobalización, erosionando a través de ese proceso la gobernanza democrática en las naciones, y los márgenes de autonomía de los gobiernos para promover sus propias políticas públicas. De los 242 casos resueltos por las instancias de solución de controversias de la OMC hasta hoy, solamente en 22 de ellos las políticas nacionales lograron prevalecer, no obstante que en muchas ocasiones los asuntos controvertidos no estaban en puridad referidos a cuestiones de comercio internacional. $\mathrm{Y}$ 
dentro de pocos días, el sistema de solución de controversias de la OMC quedará paralizado debido al bloqueo estadounidense a la nominación de nuevos integrantes para su Panel de Apelaciones.

Pero, las deficiencias del multilateralismo y del orden global en general no son meramente institucionales, sino que hunden sus raíces muy profundamente en nuestros marcos conceptuales. Las nociones de soberanía exclusiva y excluyente de los estados, la territorialidad, y diversas construcciones teóricas que reivindican la competencia de las superpotencias para afirmar su hegemonía, representan conceptualizaciones ineficaces y hasta contraproducentes para enfrentar los retos contemporáneos de la gobernanza global. A esas obsoletas categorías debemos contraponerles las emergentes nociones del derecho constitucional global, del derecho administrativo global; y el reconocimiento de la existencia de bienes y males públicos globales, cuya atención demanda la acción colectiva mundial.

$\mathrm{Y}$ es, al calor de estas emergentes categorías conceptuales, que tenemos que reformar el multilateralismo que legaremos a las nuevas generaciones. Este debe someterse a normas y mecanismos de control democrático, y debe brindar amplia cabida la participación ciudadana dentro de sus procesos de toma de decisión.

Tenemos una deuda histórica y ética con nuestro Hogar Común y con las futuras generaciones, de crear a través de un multilateralismo renovado condiciones de gobernanza global que respondan eficazmente a los retos actuales y futuros de la humanidad; que promuevan la convivencia solidaria y pacífica; que garanticen la sostenibilidad planetaria; que forjen una cultura de ciudadanía global; y que distribuyan equitativamente los frutos del bienestar que colectivamente vamos creando.

En suma, tenemos ante nosotros el desafiante reto de forjar un multilateralismo cuyo centro gravitacional sea la persona humana, y no la competencia hegemónica entre superpotencias ni el desbocado ejercicio de la soberanía estatal. Ese nuevo multilateralismo ineludiblemente tendrá que tener, para reclamarse legítimo y ser sostenible, un perfil democrático, participativo y transparente.

\section{Bibliografía}

Bailey, T. (1957, junio). Woodrow Wilson Wouldn't Yield. American Heritage, 8(4), 2025, 105-106.

Beaulac, S. (2004). The westphalian model in defining international law: Challenging the myth. Australian Journal of legal History, 8(2), 181 - 213.

Bekkers, V., Dijkstra, G., Edwards, A. y Fenger M. (2007). Governance and the Democratic Deficit. Assessing the Democratic Legitimacy of Governance Practices. Aldershot: Ashgate.

Burke, A. y Parker, R., eds. (2017). Global Insecurity. Futures of Global Chaos and Governance. London: Palgrave Macmillan.

Carr, E.H. (2016). The Twenty Years' Crisis, 1919-1939. London: Palgrave Macmillan. 
Casanovas La Rosa, O. O. (2019). En el centenario del tratado de Versalles. Revista Española de Derecho Internacional, 71(1), 17 - 22.

Clavin, P. (2013). Securing the World Economy. The Reinvention of the League of Nations, 1920-1946. Oxford: Oxford University Press.

Cox, M., Manning, D., Quah, D., Kitchen, N. y Scholefield, S. (2015). 21 ${ }^{\text {st }}$ Century Power. Dislocation, Diffusion and Decay. London: LSE Ideas.

Frey, M., Kunkel, S., y Unger C.R., eds. (2014). International Organizations and Development, 1945-1990. Houndsmill: Palgrave Macmillan.

Friedman, T. L. (2019, noviembre 26). The World-Shaking News That You're Missing. The New York Times, A: 23.

Grant, R.G. (2001). World War I. Armistice 1918. Austin: Raintree, Steck-Vaughn.

Gros, L. (1948, enero). The Peace of Westphalia (1648-1948). The American Journal of International Law, 42(1), 20-41.

Hassan D. (2006). The Rise of the Territorial State and the Treaty of Westphalia. Yearbook of New Zealand Jurisprudence, 9, 62 - 70.

Hogan, M. J. (1987). The Marshall Plan. America, Britain, and the reconstruction of Western Europe, 1947-1952. Cambridge: Cambridge University Press.

Innerarity, D. y Solana, J., eds. (2013). Humanity at Risk. The Need for Global Governance. New York: Bloomsbury.

Keynes, J.M. (2019). The Economic Consequences of Peace. With a new introduction by Michael Cox. Cham: Palgrave Macmillan.

Lowry, B. (1996). Armistice 1918. Kent: The Kent State University Press.

Luard, E. (1982). A History of the United Nations. Volume 1: The Years of Western Domination, 1945-1955. London: Macmillan.

Luard, E. (1989). A History of the United Nations. Volume 2: The Age of Decolonization, 1955-1965. New York: Palgrave Macmillan.

MacMillan, M. (2002). Paris 1919. Six Months That Changed the World. New York: Random House.

McGlinchey, S. (2010, septiembre 08). E.H. Carr and the Failure of the League of Nations. E-International Relations. Visitado octubre 12, 2019, de www.e-irinfo/2010/09/08/e-h-carr.and.the-failure-of-the-league-of-nations-a-historical-overview/

McPherson, A. $y$ Wehrli, Y, eds. (2015). Beyond Geopolitics. New Histories of Latin America and the League of Nations. Alburqueque: New Mexico University Press. 
Mingst, K.A. $y$ Karns, M.P. (2011). The United Nations in the 21st Century. Boulder: Westview Press.

Moreland, W. (2019). The Purpose of Multilateralism. A Framework for Democracies in A Geopolitically Competitive World. Washington, D.C.: Brookings.

Niemeyer, G. (1952, noviembre). The Balance-Sheet of the League Experiment. International Organization, 6(4), 537-558.

Osiander, A. (2001, primavera). Sovereignty, International Relations and the Westphalian Myth. International Organization, 55(2), $251-287$.

Pedersen, S. (2007, octubre). Back to the League of Nations. American Historical review, 112(4), $1091-1117$.

Pedersen, S. (2015) The Guardians. The League of Nations and the Crisis of Empire. New York: Oxford University Press.

Rosenbach, E. $y$ Mansted, K. (2019). The Geopolitics of Information

Cambridge: Harvard Kennedy School Belfer Center for Science and International Affairs.

Ruggie, J.G. (1992, verano). Multilateralism: the Anatomy of an Institution. International Organization, 46(3), 561-598.

Schain, M., ed. (2001). The Marshall Plan: Fifty Years After. New York: Palgrave.

Steil, B. (2018). The Marshall Plan. Dawn of the Cold War. New York: Simon \& Schuster.

Steiner, Z. (2005). The Lights That Failed. European International History 1919-1933. New York: Oxford University Press.

Straumann, B. (2008). The Peace of Westphalia as a Secular Constitution. Constellations, $15(2), 173-188$.

Weinberg, G. L. (1995). A World at Arms. A Global History of World War II. New York: Cambridge University Press.

Weiss, T.G. (2015). The United Nations: before, during and after 1945. International Affairs, 91(6), 1221-1235.

White, N.D. (2019). The legacy of the League of Nations: Continuity or change. Revista Española de Derecho Internacional, 71(2), 277-284. 